\title{
Premature development of erosive osteoarthritis of hands in patients with chronic renal failure
}

\author{
I J S Duncan, N P Hurst, R Sebben, S C Milazzo, A Disney
}

Rheumatology Unit and *Renal Unit, The Queen Elizabeth Hospital, Woodville, South Australia 5011 I J S Duncan N P Hurst

S C Milazzo A Disney

Department of Radiology, The Queen Elizabeth Hospital, Woodville,

South Australia 5011

R Sebben

Correspondence to:

Dr N P Hurst,

Rheumatology Unit,

Northern General Hospital

Ferry Road, Edinburgh

IH5 2DQ.

Accepted for publication 21 July 1989

\begin{abstract}
The prevalence of grade III or IV osteoarthritis was determined in 210 patients with chronic renal failure, of whom 94 were receiving chronic haemodialysis and 116 had functioning renal transplants. The prevalence of grade III or IV osteoarthritis was three times greater in patients under 65 than in a control population, and all but two affected patients also had erosion of subchondral bone in at least one affected joint. The excess of osteoarthritis was apparent in both the transplant recipients and those receiving haemodialysis. Over the age of 65 there was no significant difference in prevalence. Metabolic bone disease, including osteopenia, might contribute to the development of erosive osteoarthritis in chronic renal failure.
\end{abstract}

We recently reported two cases, one a patient receiving chronic haemodialysis and the other a
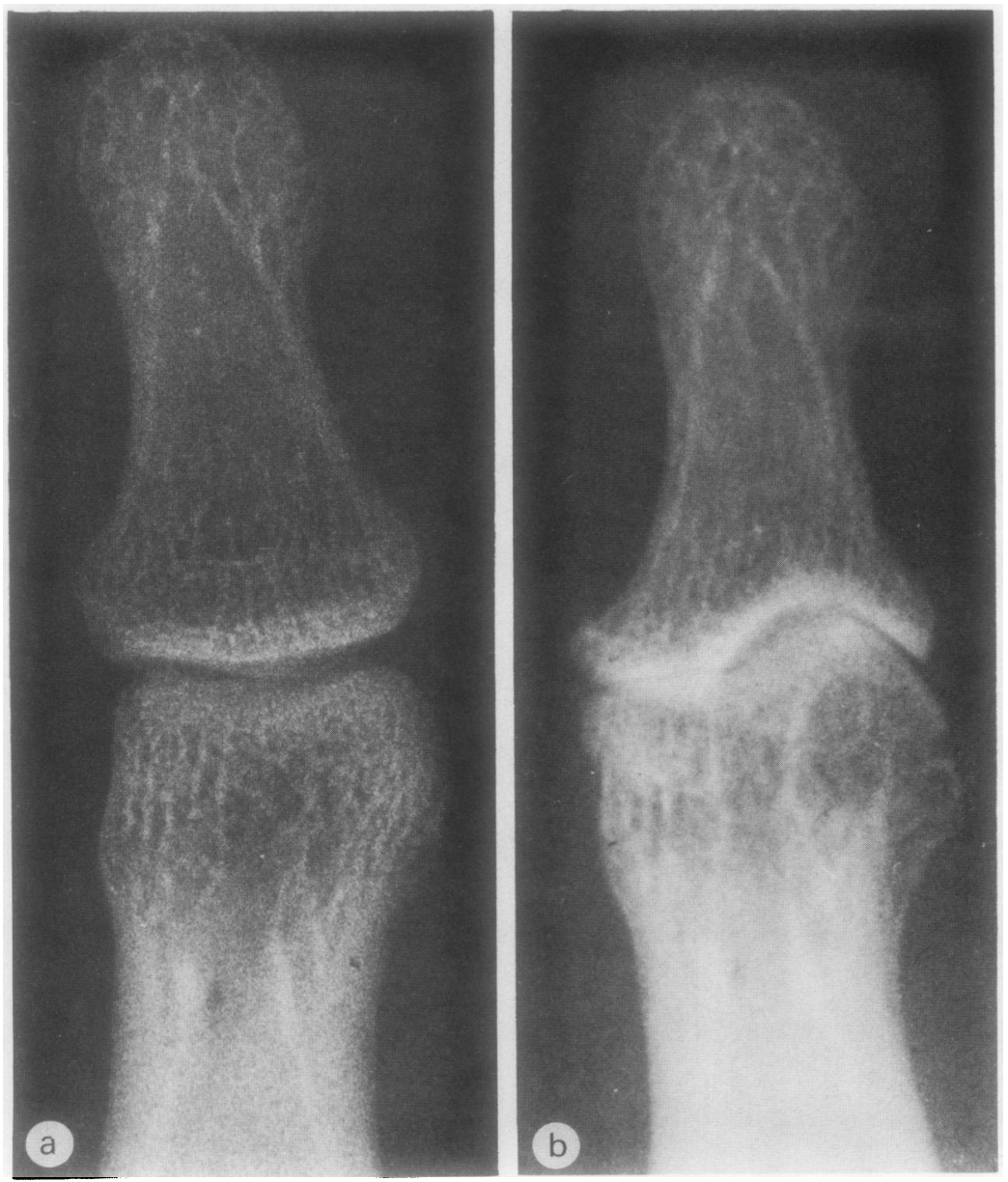

renal transplant recipient, who had developed severe progressive erosive osteoarthritis $(\mathrm{OA})$ of the hands before the age of $35 .^{1}$ Previous studies of patients receiving chronic haemodialysis have noted degenerative changes in the small joints of hands but have not established whether this is in some way related to chronic renal failure or is simply related to age. ${ }^{2-9}$ There have been no comparable studies of renal transplant recipients. We have therefore conducted a survey of patients receiving chronic haemodialysis and renal transplant recipients at the Queen Elizabeth Hospital to establish the prevalence, age distribution, and clinical features of grade III and IV $\mathrm{OA}$ of the hands in patients with chronic renal disease.

Patients and methods

Ninety four ( 55 male, 39 female) of a possible 118 patients receiving chronic haemodialysis were surveyed between May 1986 and January 1987; this group has been described elsewhere. ${ }^{2}$ Of a possible 188 renal transplant recipients, 116 ( 74 male, 42 female) seen consecutively at the renal outpatient clinic between April and June 1988 were also studied. Patients who received renal allografts between the time of the survey of dialysis and transplant recipients were included only in the group receiving chronic haemodialysis. Five transplant recipients declined to participate. All patients underwent anteroposterior radiographs of hands and were questioned about the presence of pain and stiffness in the fingers. Radiographs were screened by RS for the presence of OA. Abnormal radiographs were reviewed in a blinded fashion by IJSD and NPH and graded 0 to IV for OA according to the method of Lawrence and Kellgren ${ }^{10}$; those patients with radiological grade III or IV OA in at least one joint were selected for further study. Grade III OA comprises moderate joint space narrowing and definite osteophytes, whereas grade IV comprises complete loss of joint space with florid osteophytes and subchrondral sclerosis.

Figure 1: Radiographs of the distal interphalangeal joint of the left middle finger of a female transplant recipient

(17 years' duration; no haemodialysis) at age $29(a)$ and 37 (b), showing progressive loss of articular cartilage, erosion of subchondral bone, and osteophytosis. Similar radiological changes in different stages of evolution were present in other distal interphalangeal joints. Several fingers in both hands were affected by Heberden's nodes, and a cystic node had occurred without inflammation over the distal interphalangeal joint of the left little finger at age 36. All distal interphalangeal joints were stiff and restricted. 
Prevalence of grade III and IV osteoarthritis in hands of patients with chronic renal disease. Number (\%) of patients is given

\begin{tabular}{lllllll}
\hline & \multicolumn{1}{l}{ Age (years) } & & & \\
\cline { 2 - 7 } & $15-24$ & $25-34$ & $35-44$ & $45-54$ & $55-64$ & $\geqslant 65$ \\
\hline Controls & $1(0 \cdot 3)$ & $1(0 \cdot 3)$ & $3(0 \cdot 7)$ & $14(3)$ & $34(10 \cdot 5)$ & $115(29 \cdot 7)$ \\
All renal patients & $0(0)$ & $1(3 \cdot 5)$ & $3(5 \cdot 9)$ & $3(6 \cdot 3)$ & $11(19 \cdot 3)$ & $3(15 \cdot 8)$ \\
Patients receiving CHD & $0(0)$ & $1(10)$ & $1(5 \cdot 6)$ & $1(5 \cdot 6)$ & $8(25 \cdot 8)$ & $2(13 \cdot 3)$ \\
Transplant recipients & $0(0)$ & $0(0)$ & $2(6 \cdot 1)$ & $2(6 \cdot 7)$ & $3(11 \cdot 5)$ & $1(25)$ \\
\hline
\end{tabular}

${ }^{*} \mathrm{CHD}=$ chronic haemodialysis.

Those patients with the additional feature of erosion of subchondral bone were designated as having erosive $\mathrm{OA}$.

Control data were obtained from the Leigh and Wensleydale survey. ${ }^{11}$ The prevalences of grade III or IV OA in the distal interphalangeal joints in that study were used as our control values. Statistical analyses were performed with
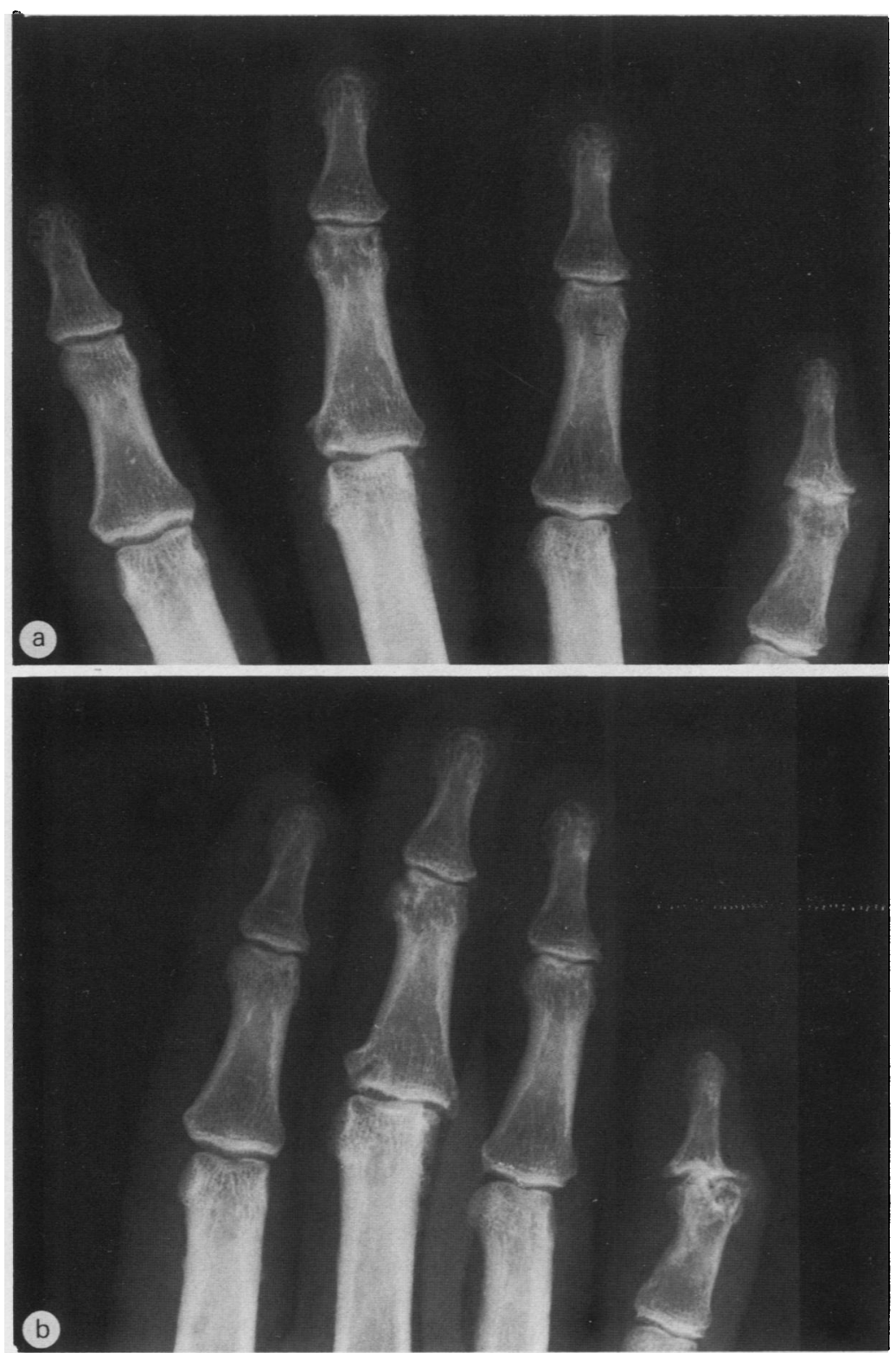

Figure 2: Radiographs of fingers of the right hand of a female transplant recipient (15 years' duration; one year haemodialysis) at age $53(a)$ and $58(b)$. Note progression of arthritis, resulting in severe loss of subchondral bone, osteophytosis, and deformity in the distal interphalangeal joint of the little finger, and loss of articular cartilage, subchondral sclerosis, osteophytosis, and deformity in the interphalangeal joint of the middle finger. Less severe osteoarthritis is present in other distal interphalangeal joints. the $\chi^{2}$ test (with Yates's correction for small numbers).

\section{Results}

The prevalence of grade III or IV OA amongst the renal patients (chronic haemodialysis and transplant recipients combined) aged 15-64 years $(9 \cdot 4 \%)$ was significantly higher than in the normal population $(3 \%)(\mathrm{p}<0.001)$. Over the age of 65 , however, the number of renal patients available for study was small (19 total), and in this group there was no significant difference in prevalence of OA compared with controls. The sex ratios (female to male) in the transplant recipients, those receiving chronic haemodialysis, and controls were $2.9,1.64$, and 1.86 respectively.

Analysis of prevalence by decennial cohorts (24-34; 35-44; 45-54; 55-64; >65 years) showed that compared with the control data there was an excess of grade III or IV OA among patients with renal disease in each cohort up to the age of 65 . This excess was apparent in both the patients receiving chronic haemodialysis $>25$ years and the transplant recipients $>35$ years (table). The differences between the renal patients and control data reached significance in the age cohorts $35-44(p<0.05)$ and 55-64 ( $<<0.05)$.

Of the 21 patients with grade III or IV OA, all had distal interphalangeal joint involvement, and in addition five and nine respectively had proximal interphalangeal joint and first carpometacarpal joint involvement. All but two of the 21 patients also had erosion of subchondral bone in at least one of the affected joints. In most patients the joints were affected in an asymmetrical fashion with articular abnormalities ranging from normal in some joints to severe destructive changes in others. Review of earlier hand radiographs showed that minor osteoarthritic changes often progressed rapidly to destructive erosive OA (figs 1-3), a phenomenon which we had observed previously in our index cases." No patients had degenerative changes isolated to the proximal interphalangeal or first carpometacarpal joints. In 17 of these 21 patients Heberden's nodes were clinically apparent, and three of the five with proximal interphalangeal joint involvement had Bouchard's nodes. None of the patients gave a definite history of an inflammatory episode involving affected joints, but 14 of the 21 affected had joint pain or stiffness with mild to moderate loss of function. Several patients had marked vascular calcification, but significant periarticular soft tissue calcification was present in only one patient with OA. 

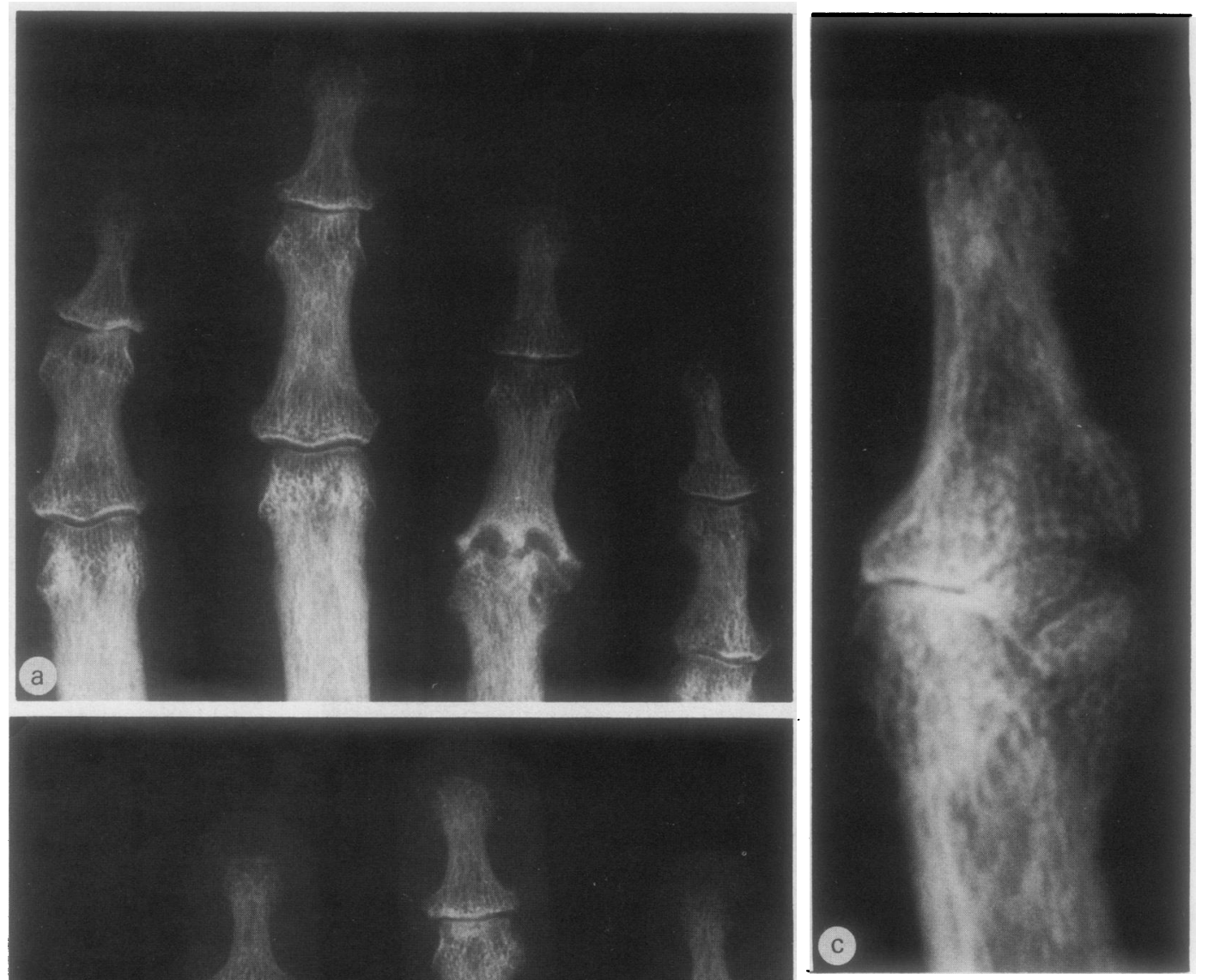

Figure 3: Radiographs of fingers of the right hand (a), left hand (b), and right thumb (c) at ages 59 (series I) and 63 (series II, facing page) of a patient receiving chronic haemodialysis (five years' haemodialysis; no transplant). Note the progression of arthritis in the thumb, resulting in severe resorption of subchondral bone and deformity. Progressive arthritic changes have occurred in various interphalangeal joints of fingers from age 59 to 63, with loss of articular cartilage, subchondral sclerosis, osteophytosis, and, eventually, resorption of subchondral bone. Note that changes are in different stages of evolution in different fingers.

\section{Discussion}

In this study our aim was to determine whether there is an increase in prevalence of grade III or IV OA of hands in chronic renal failure and whether the condition is accelerated and occurs at an earlier age. Our data suggest that although there is no overall increase in prevalence of grade III or IV OA, it does occur at an earlier age in patients with chronic renal failure and shows a marked propensity to progress to erosion of subchondral bone. Furthermore this phenomenon is apparent both in patients receiving chronic haemodialysis and in transplant recipients. The radiological appearances are indistinguishable from those associated with so called 'erosive OA', but our patients did not report inflammatory symptoms or signs in affected joints. One transplant recipient who developed a typical cystic Heberden's node had no associated inflammation. The usual inflammatory changes might have been suppressed by her corticosteroid treatment.

An important question is whether our use of the Leigh and Wensleydale study for our control data is valid. The radiographic criteria used were the same, but the population from which that sample was taken may not be comparable with our regional referral population for which no equivalent data are available. A recent survey from Holland found a prevalence of grade III or IV OA in distal interphalangeal joints of hands of $3.8 \%$ in men and $5.9 \%$ in women aged 45-64 years. ${ }^{12}$ In the Leigh and Wensleydale survey the prevalences were in fact slightly higher $-4 \cdot 7 \%$ of men and $7 \cdot 4 \%$ of women. Higher prevalences of $\mathrm{OA}$ have been 

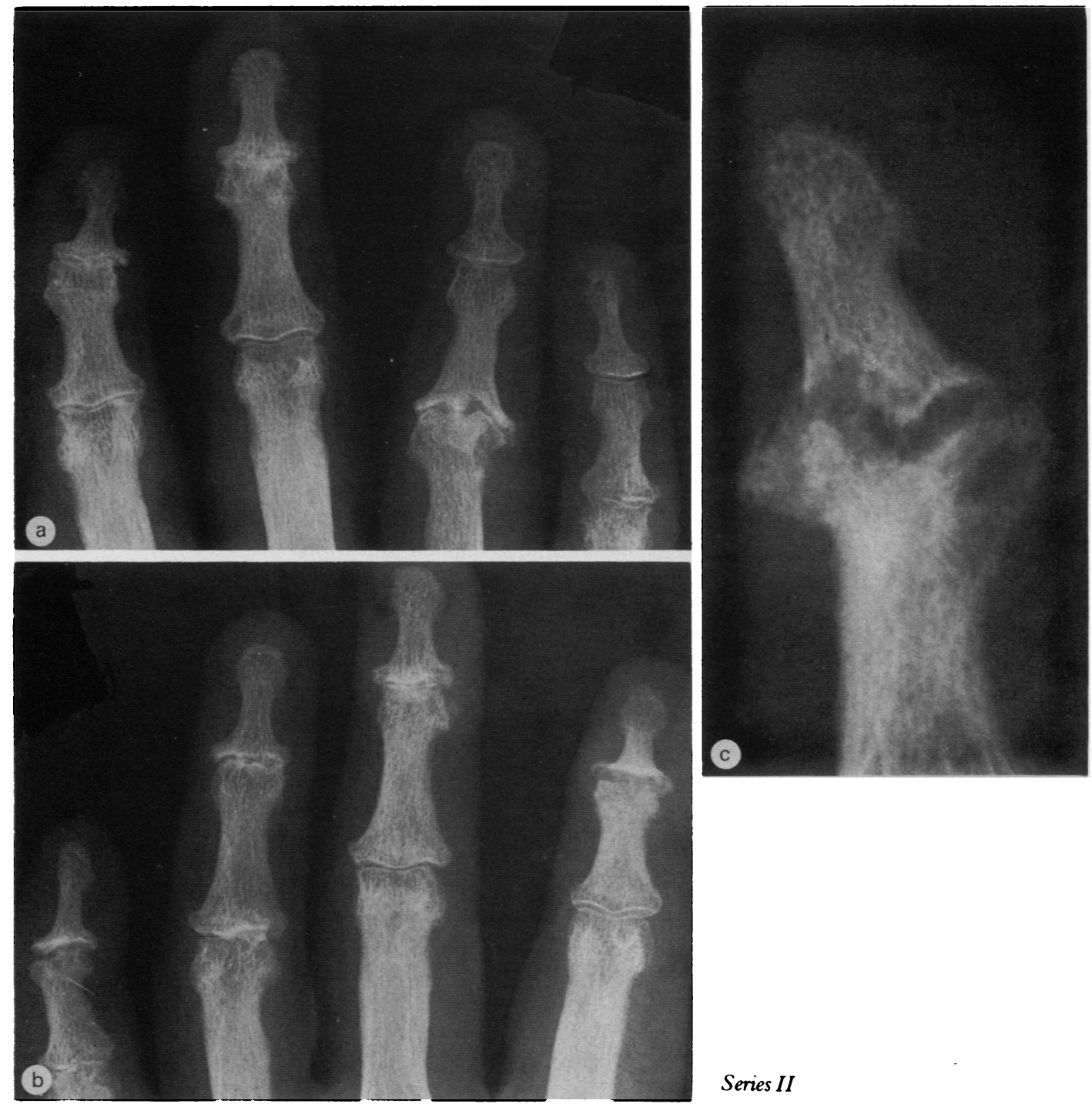

Series II

noted in Pima and Blackfeet Indians and in one Japanese group. ${ }^{13}$ Most patients in our study were of Northern European descent, however, and it is unlikely that our referral population had a substantially different prevalence of OA from the United Kingdom or Dutch populations.

Interpretation of radiological changes in the finger joints of patients with chronic renal failure has been controversial. ${ }^{1}$ Sundaram et al described erosive changes affecting the distal interphalangeal or metacarpophalangeal joints in 24 of 80 patients receiving chronic haemodialysis. ${ }^{3}$ In some patients these were associated with degenerative changes resembling erosive $\mathrm{OA}$, but exact numbers or ages of patients were not listed. Rubin et al in a study of 59 patients receiving chronic haemodialysis showed a similar prevalence of small joint erosions and noted 'coincidental' OA in small joints of the hands or knees in $24 \%$ of their patients. ${ }^{4}$ Naidich et al reported a radiological survey of 81 patients receiving chronic haemodialysis and identified 32 with severe 'erosive osteoarthropathy' affecting equally distal interphalangeal, proximal interphalangeal, and first carpometacarpal joints. ${ }^{5}$ Affected joints were grossly narrowed with the gull's wing appearance and lateral subluxation typical of erosive OA. ${ }^{14}$ Surprisingly, Naidich concluded that the changes were not typical of OA because of the presence of osteopenia, cystic change, and sometimes soft tissue calcification. Goldstein $e t$ $\mathrm{al}^{7}$ also described 11 patients receiving chronic haemodialysis who had a chronic hand arthropathy with similar radiographic features but noted an absence of 'spurring'-that is, osteophytes, and thus also concluded that the condition is distinct from OA. In our study we specifically identified those patients with radiological grade III or IV OA, who by definition have prominent osteophytes, and our data confirm that there is an association between chronic renal failure and premature onset of severe OA. In addition, almost all our patients showed evidence of progression to erosion of subchondral bone typical of erosive OA.

Most authors have not commented on whether the above radiological changes result in functional loss, and only two reports suggest that significant symptoms or deformity may develop. ${ }^{47}$ We have found OA in the hands to be a significant source of symptoms and functional loss in patients with chronic renal failure. 
A similar conclusion was drawn in a recent survey of dialysis related arthropathy undertaken in this hospital, which found that $\mathrm{OA}$ is a common cause of small joint stiffness and pain. ${ }^{2}$ Our study also extends this finding to transplant recipients.

The pathogenesis of articular damage in chronic renal failure remains speculative. ${ }^{1}$ The concept suggested by Bywaters et al that erosions may result from collapse of softened subchondral bone at sites of mechanical pressure may be important in patients with chronic renal failure. ${ }^{15}$ Sundaram et al also concluded that this was a likely mechanism of articular damage in patients receiving chronic haemodialysis. ${ }^{3}$ The progression from minor osteoarthritic changes to marked bone erosion observed in our patients is consistent with Bywater's hypothesis. Thus metabolic bone disease, including osteopenia, ${ }^{16}$ which is multifactorial and ubiquitous in chronic renal failure, might contribute to the development of erosive $\mathrm{OA}$ in patients receiving chronic haemodialysis and in transplant recipients.

We conclude that chronic renal failure is a risk factor for the premature development of grade III or IV OA and that this risk remains even after successful renal transplantation. Furthermore, these patients also develop progressive erosion of subchondral bone typical of erosive OA. Metabolic factors associated with chronic renal failure are presumed to be responsible. Our findings may be relevant to the understanding of the pathogenesis of nodal and erosive $\mathrm{OA}$ in the normal aging population.
1 Duncan I J S, Hurst N P, Disney A, Sebben R, Milazzo S C. s chronic renal failure a risk factor for the development of erosive osteoarthritis? Ann Rheum Dis 1989; 48: 183-7.

2 Hurst N P, van den Berg R, Disney A, et al. Dialysis related arthropathy: a survey of 95 patients receiving chronic arthropathy: a survey of 95 patients receiving chronic haemodialysis with special reference to $\beta_{2}$ microglob 3 Sundaram M, Wolverson M K, Heiberg E, Grider R D. Erosive azotaemic osteodystrophy. A7R 1981; 136: 363-7.

4 Rubin L A, Fam A G, Rubenstein J, Campbell J, Saiphoo C. Erosive azotaemic arthropathy. Arthritis Rheum 1984; 27 1086-94.

5 Naidich J B, Karmel M I, Mossey R T, Bluestone P A, Stein $H$ L. Osteoarthropathy of the hand and wrist in patients undergoing longterm haemodialysis. Radiology 1987; 164 205-9.

6 Resnick D L. Erosive arthritis of the hand and wrist in hyperparathyroidism. Radiology 1974; 110: 263-9.

Goldstein S, Winston E, Chung T J Shreekant C Pariser K. Chronic arthropathy in long-term haemodialysis. Am $7 \mathrm{Med}$ 1985; 78: 62-86.

8 Brown E A, Arnold I R, Gower P E. Dialysis arthropathy: complication of long term treatment with haemodialysis. $\mathrm{Br}$ Med F 1986; 292: 163-6.

9 Hamilton S, Knickerbocker W J. Peri-articular erosions in the hands and wrists in haemodialysis patients. Clin Radiol 1982; 33: 19-24.

10 Lawrence J S, Kellgren J H. Radiological assessment of osteoarthrosis. Ann Rheum Dis 1957; 16: 494-502.

11 Lawrence J S, Bremner J M, Bier F. Osteoarthrosis prevalence in the population and relationship between symptoms and $\mathrm{x}$-ray changes. Ann Rheum Dis 1966; 25 $1-24$.

12 van Saase J L C M, Vandenbroucke J P, Romunde L K J van Valkenburg H A. Osteoarthritis and obesity in the general population. A relationship calling for an explanation. $f$ population. A relationship

13 van Saase J L C M, van Romunde L K J, Cats A, Vandenbroucke J P, Valkenburg H A. Epidemiology of osteoarthritis: Zoetermeer survey. Comparison of radiological osteoarthritis in a Dutch population with that in 10 other populations. Ann Rheum Dis 1989; 48: 271-80.

14 Ehrlich G E. Erosive inflammatory and primary generalized osteoarthritis. In: Moskowit\% R W, Howell D S, Goldberg V M, Mankin $\mathrm{H}$ J, eds. Osteoarthritis-diagnosis and management. Philadelphia: Saunders, 1984: 201-4.

15 Bywaters E G L, Dixon A St J, Scott J T. Joint lesions of hyperparathyroidism. Ann Rheum Dis 1963; 22: 171-87.

16 Smythe H A. Osteoarthritis, insulin, and bone density. f Rheumatol 1987; 14 (suppl): 91-3. 